Published as: Griffiths, M.D. (2011). Gaming convergence: Further legal issues and psychosocial impact. Gaming Law Review and Economics, 14, 461-464.

Gaming convergence: Further legal issues and psychosocial impact

\author{
Dr Mark D. Griffiths \\ Professor of Gambling Studies \\ International Gaming Research Unit \\ School of Social Sciences \\ Nottingham Trent University \\ Burton Street \\ Nottingham \\ NG1 4BU \\ United Kingdom
}

Disclaimers and Competing Interests: The author did not receive any funding for this article. The author has received funding for a number of research projects from the Gambling Commission of Great Britain and the UK Responsible Gambling Fund. The author also undertakes consultancy for various gaming companies in the area of social responsibility in gambling. 


\section{Gaming convergence: Further legal issues and psychosocial impact}

The editorial by Owens ${ }^{1}$ in a recent issue of Gambling Law Review and Economics raised a number of interesting questions in relation to the convergence of gambling and gaming. The uptake and use of games in associated leisure contexts such as gambling indicates the level of interest in game-based activities. In general, the flexibility of the medium, the wide application of the formats, and the immersive and interactive qualities have generated interest in using game technologies for many purposes, including educational activities and leisure-based activities (e.g., gambling). The crossover between games and mobile devices, including smart phones, MP3 players, and handheld game consoles, is becoming a topic of eager interest in a variety of contexts. Mobile hardware has been seen as a device waiting for an application. Gambling and gaming offer high levels of engagement and content generation that could be imported fairly easily onto these kinds of devices. Furthermore, there are many examples of the use of games and mobiles being piloted in a range of contexts. ${ }^{2}$

Gamers are 'techno-savvy' to begin with, and are likely to be less cautious with new forms of technology. Remote gaming is anonymous, and can provide immediate gratification, anytime, anywhere. The limiting aspects of the technological and protocol demands of mobile gaming (graphics, sound, and displays) are largely in the process of being resolved. The convergence between gambling, games, and emerging technologies — such as mobile and interactive television-has considerable scope for development, with the reach of mobile gambling and gaming offering a significant market, as well as the potential for crossover with new interfaces, such as "augmented reality." This will provide greater flexibility in terms of promoting gaming experiences away from the desktop computer. If the fastest innovations are in areas of overlap between different media, the potential for future gambling and gaming opportunities may continue for a long while to come.

In some countries, video game technology has been used in gambling products for a number of years (such as some of Loto Quebec's use of video game technology in lottery products in Canada). One interesting development is that video game players are now being paid to kill within gaming environments. ${ }^{3}$ On one level, this can be seen as the next phase in the evolution of gaming, as game developers are constantly looking for new ways to increase revenue. Since 2006, a number of servers aimed at the adult gaming 
market have launched services that pay video game players every time they kill within the game they are playing. In some ways, this activity is akin to some types of online gambling, such as online poker. As Harper (see footnote 3) points out, the prospect of gaining revenue "from playing video games makes online poker seem as old-fashioned as its physical equivalent."

One of the legal implications of being paid to kill within the confines of a computer game is that the activity is defined as a skill-based (as opposed to a chance-based) activity, and is therefore exempt from the regulations set down in many countries' gambling regulations. It is likely that more and more gambling companies will start to utilise video game technology within their products, and this will then become an issue that gambling regulators around the world will have to re-examine in terms of the gambling legislation.

One of the problems within online computer gaming is that cheating can be common. Harper highlights the case of a "semi-professional gamer" who played Tournament.com for several months, but stopped playing this gambling-type computer game because he claimed other players had installed third party computer programmes to help them to play well (and win more money as a consequence). The player reported to Harper that "it would take more than a month before (the gaming company) actually caught a cheater who ended up raking thousands of dollars from other gamblers" (p.3). Tournament.com has now ceased trading, but other gaming companies (such as kwari.com) have set up similar first-person shooter games and learning the lessons from those sites where allegations of cheating occurred. On the Kwari.com website it says:

"Kwari has been designed with a singular purpose in mind - to give you the opportunity to translate your shooter skills into some serious cash. Everything about the game bas been tailored to that effect, so much so that even an average player should be able to get abead in the game quickly. Every time you bit another player in Kwari you make money. Every time you are hit by another player it costs you. Every shot counts. How much is down to the stake level you play in. But this is not the only way to win. Doing damage to yourself, breaking crates, use of certain map features or picking up additional weapons, pickups and health packs may have a fractional cost attached. This cost is transferred between a series of jackpots, prizes and awards available in the game, all of which can be won by any player, regardless of the skill or stake level of game they prefer to play. At no point, however does Kwari take any of this money. 100\% of the cash generated through playing the game goes back to the players in the form of prizes. The most frequent of these prizes is the Pill Jackpot, which is split between the player who carries the Pill the longest, and the player who finishes the round with the Pill. Other jackpots in the game are won by collecting Kwari keys which spawn regularly in the game until the prize has been won. Depending on the length of the key series required to win, different jackpots will pay out over different periods, 
ranging from one hour to six months, and once a jackpot has been won all the key chains for that prize start again.”

Kwari.com makes their money via subscriptions to play, and in that way, is very similar to online poker sites that take a very small commission on winnings. Kwari.com says it wants to appeal to casual players who normally use online poker sites. It also claims that it wants people to play the game for no more than an hour, two times a week, and say they will put a cap on the amount a user can spend a month unless they have been vetted as suitable for their high-stakes tournament. These types of actions are akin to the social responsibility practices found in more traditional online gambling sites, and emphasise that these types of game are really forms of gambling in all but name. Many gaming companies will be observing whether sites like Kwari.com succeed financially. If it turns out to be a success, a lot of the bigger computer game companies will want to get in on the act, which could change the way people game online.

To flourish in an evolving digital landscape, television companies are also formulating strategies for targeting particular segments of the industry or market. Platform operators appear to be deploying consumer-driven applications, such as gambling and gaming. They are creating an environment where content originators and channel operators can innovate and profitably create interactive broadband content. Interactive television is seen as a way of rapidly expanding gaming and gambling, because of its naturalness and ease of use. This interest in all things interactive directly results from growing competition between Internet service providers, satellite TV, and cable-TV outfits. All these different providers are looking for features to differentiate themselves. One of the most potentially lucrative vehicles for this has been interactive television gaming and gambling.

Clearly convergence is playing an informing and reinvigorating role in gambling and gaming and beginning to subvert 'traditional' gambling forms. While there are many advantages of convergence in a wide variety of contexts, there may be some disadvantages for those who use convergent technology excessively. However, the potential downsides need to be examined in the overall context. ${ }^{4}$

The rise and challenges of gambling and gaming cannot be seen in isolation, particularly as there is ever-increasing multi-media integration between the Internet, mobile phones, 
and interactive television (i-TV). On a commercial level, it may be that people are more likely to spend money in particular interactive media. For instance, Griffiths ${ }^{5}$ noted that the Internet can be described as a "lean forward" medium. This means that the user (who is usually alone) takes an active role in determining what they do. Computers are better at displaying text than television, and have a wider range of fine-tuning controls through the mouse and keyboards. This makes them more suitable for complex tasks such as obtaining insurance quotations or travel itineraries. In contrast, the television is a 'lean back' medium where the viewer (often as part of a group) is more passive and seeks less control over what is going on. The television is better at displaying moving images than computers or mobile phones. This may have implications for the types of gaming done in particular media.

It has also been argued (see footnote 5) that interactive television may also help in one other important area-trust. People appear to trust their television even though it is accessing the Internet in the same way as a computer. However, as argued above, i-TV is a "lean back" service. If a person is relaxed, sitting back on their sofa, it will make television the key to creating a true mass market for online commercial activity (including gambling and gaming). In addition, some i-TV services can be linked to actual television programmes. Browsing and playing games by i-TV are still in their infancy, but look set to expand significantly in the future.

Added to this is the potential of social networking sites to normalise gambling behaviours as part of the consumption patterns of a non-gambling leisure activity. This may change social understandings of the role of gambling amongst young people. ${ }^{6}$ Downs $^{7}$ also reported a type of pseudo-gambling among the social networking application "Fluff Friends" that has over 100,000 active users per month. In this social networking forum, users (typically young girls) create Fluff'Art. To do this they have to earn "munny" (sic) - a type of virtual money through pet racing. Pet racing costs 1-point per race and winnings can be up to 4000 points. Clearly, there is no money changing hands, but young children are learning the mechanics of gambling, and Downs asserts there are serious questions about whether gambling with virtual money encourages positive attitudes towards gambling in young people. For instance, does gambling with virtual money lead to an increased prevalence of actual gambling?

Other social networking games like Farmville have also been argued to have similarities to 
gambling. ${ }^{8}$ In March 2010, the British press reported the case of a 12-year-old boy who spent $£_{6} 625$ on his mother's credit card and $£_{2} 288$ of his own savings playing FarmVille without the knowledge of his parents. The mother tried to get a refund from her credit card company. However, they advised her that she could only get one if she reported her son to the police and obtained a crime number. The family was forced to pay the money, as the 12-year old boy lived at his parents' house. Facebook has since disabled the boy's account, and the company who created the game (Zynga) simply advised the boy's parents to use password protection on house computers in the future.

The mother has admitted that her son was at fault, but claims that the main stakeholders (Facebook, Zynga, and her credit card company, HSBC) should take some responsibility in situations such as this one and implement systems to stop incidents like this from happening again (such as more thorough age verification procedures). Other critics argue that games like Farmville should not be 'monetised' to raise additional revenue for the game's owners and operators. Interestingly, the credit card company said that if the card had been used on an internet gambling site, the company would have investigated the incident as an example of unusual usage. However, in this case, the purchasing of $£ 625$ of Facebook credits in a two-week period was not viewed as an example of unusual usage.?

The rise and challenges of convergent gambling and computer gaming cannot be seen in legal isolation, given the ever-increasing multi-media integration. Commercial operators always appear to exploit new market opportunities in emerging media, and are often two steps ahead of contemporary legislation. Convergent gambling and gaming is of potential interest to many stakeholder groups, including researchers and the gaming industry. Research needs to be carried out on both the potential advantages (e.g., the increased fun and excitement for the players; increased revenues for the gaming operators) and potential disadvantages (e.g., the psychosocial impact of excessive use).

\section{References}

(1) Owens, M., Is i-gaming Leaving Gambling Behind? New social Media Vary the Formats and Business Models. 14 Gambling Law REviEW AND ECONOMICs, 14, 737-739 (2010). 
(2) Griffiths, M.D., Mobile Phone Gambling, in D. Taniar (Ed.), Encyclopedia OF Mobile Computing And Commerce, pp.553-556 (2007). Pennsylvania: Information Science Reference.

(3) Harper, A., "Pay-per-kill shooters combine online gambling with gaming," The Guardian (Technology Supplement), November 22, 2007, p.3.

(4) de Freitas, S. \& Griffiths, M.D., The Convergence of Gaming Practices With Other Media Forms: What Potential for Learning? A Review of the Literature, 33 LEARNING, Media AND TeChNOLOGY 11-20 (2008).

(5) Griffiths, M.D., Internet Gambling: Issues, Concerns and Recommendations, 6 CYBERPsyCHOLOGY AND BEHAVIOR, 557-568 (2003).

(6) King, D.L., Delfabbro, P.H. \& Griffiths, M.D., The Convergence of Gambling and Digital Media: Implications for Gambling in Young People, 26 Journal OF GAMBLING STUDIES, 175-187 (2010).

(7) Downs, C. (2008, September), “The Facebook Phenomenon: Social Networking and Gambling." Paper presented at the Gambling and Social Responsibility Forum Conference, Manchester Metrolpolitan University, Manchester.

(8) Griffiths, M.D., Gaming in Social Networking Sites: A Growing Concern? 9(5) WORLD OnLINE GAMBLING LAW REPORT 12-13 (2010).

(9) $I d$. 